\title{
BMR
}

\section{Combination of IL-6, IL-10, and MCP-1 with traditional serum tumor markers in lung cancer diagnosis and prognosis}

Y.W. Pan ${ }^{1}$, Z.G. Zhou ${ }^{1}$ M. Wang ${ }^{1}, J$ Q P.J. $\mathbf{L v}^{2}$ and J.B. Gao ${ }^{2}$

${ }^{1}$ Department of Imaging medicine and nuclear medicine, The First Affiliated Hospital of Zhengzhou University, Zhengzhou, Henan, China ${ }^{2}$ Department of Radiology, The First Affiliated Hospital of Zhengzhou University, Zhengzhou, Henan, China

Corresponding author: J.B. Gao

E-mail: jianbogao123@sina.com

Genet. Mol. Res. 15 (4): gmr15048949

Received July 6, 2016

Accepted August 26, 2016

Published November 3, 2016

DOI http://dx.doi.org/10.4238/gmr15048949

Copyright (C) 2016 The Authors. This is an open-access article distributed under the terms of the Creative Commons Attribution ShareAlike (CC BY-SA) 4.0 License.

\begin{abstract}
Early detection and treatment is critically important for lung cancer patients. Inflammatory mediators such as IL-6, IL-10, and MCP-1 participate in lung cancer regulation. CEA, CA125, and ProGRP are commonly used serum tumor markers for lung cancer. In this study, we assessed the sensitivity and specificity of CEA, CA125, and ProGRP when used in combination with IL-6, IL-10, and MCP in lung cancer diagnosis. Serum from three different groups (healthy controls, individuals with high risk for lung cancer, and lung cancer patients) was collected. Electrochemiluminescence was used to detect expressions of CEA, CA125, and ProGRP; ELISA was used to examine serum levels of IL-6, IL-10, and MCP-1. Specificity and sensitivity of single as well as combination markers in lung cancer diagnosis were
\end{abstract}

Genetics and Molecular Research 15 (4): gmr15048949 
determined. Results indicated that CEA, CA125, ProGRP, and MCP-1 were significantly up-regulated in lung cancer patients as compared to those in controls and high risk individuals. Higher IL-6 and IL-10 levels were observed in both lung cancer patients and high-risk individuals as compared to those in controls. Highest sensitivity $(95.2 \%)$ in cancer diagnosis was achieved when all six markers were used. This was followed by a combination of IL-6, IL-10, CEA, CA125, and ProGRP (92.6\%). The most sensitive $(88.6 \%)$. Four-marker combination was composed of IL-6, CEA, CA125, and ProGRP. As the combined usage of CEA, CA125, ProGRP, IL-6, IL-10, and MCP-1 significantly improved sensitivity of lung cancer detection; this biomarker arrangement may be beneficial for early diagnosis, treatment, and prognosis of lung cancer.

Key words: Lung cancer; Serum tumor markers; Lung cancer screen; Diagnosis

\section{INTRODUCTION}

Lung cancer is a malignancy of the respiratory system, with the highest incidence and mortality rates among all malignancies (Reddy et al., 2014; Yang et al., 2014). The global mortality rate of lung cancer is rapidly rising; currently, China has the highest incidence and mortality rates of lung cancer, with an increasing number of younger patients being affected as compared to the past 20 years (Bhora et al., 2014; Burns and Stabile, 2014; Genestreti et al., 2014). The onset and development of lung cancer are driven by multiple factors, which are the result of various signaling pathways controlled by an array of genetic mutations. Therefore, it is difficult to develop rapid and accurate methods for early diagnosis of lung cancer, which leads to poor treatment outcomes in advanced disease stages (Hu et al., 2009; Northcott et al., 2009). Due to its high malignancy and rapid development, it is critical to develop methods for early detection, diagnosis, and treatment of lung cancer.

Tumor markers in the serum can reflect the onset, development, and changes in tumors, and are commonly used in screening, diagnosis, treatment evaluation, recurrence monitoring, and prognosis of cancers (Pang et al., 2009; Shi et al., 2015). One such example is carcino-embryonic antigen (CEA), which exists in trace amounts in healthy human serum. Its expression has been shown to be elevated in a variety of tumors, and is thus used as a clinical indicator for a variety of cancers (Fiala et al., 2016). In addition, while carbohydrate 125 (CA125) is undetectable in the ovaries of healthy people, it is abnormally high in patients with tumors (Ma et al., 2015). Progastrin-releasing peptide (ProGRP) is present only in the brain and gastrointestinal nerve fibers of healthy people. However, in patients with small cell lung cancer, it is released into the blood (Qader et al., 2014). As a result, plasma levels of CEA, CA125, and ProGRP are currently the most common indicators for non-small cell lung cancer. However, the sensitivity and specificity of these three indicators are inadequate for early diagnosis of lung cancer. Recently, inflammation has been proposed to be highly associated with lung cancer (Chen et al., 2015b), and the inflammatory factors IL-6 and IL-10, as well as the chemokine MCP-1, have been shown to be associated with lung cancer (Lan et al., 2015; Shinke et al., 2015). In this study, we assessed the feasibility of using traditional plasma tumor markers together with IL-6, IL-10, and MCP-1 in the screening and diagnosis of lung cancer.

Genetics and Molecular Research 15 (4): gmr15048949 


\section{MATERIAL AND METHODS}

\section{Patient information}

Lung cancer patients (142), who were diagnosed via histological examinations and had undergone surgery, were recruited from the First Affiliated Hospital of Zhengzhou University between January 2015 and December 2015. Among these patients, 72 were male and 70 were female. The average age of the patients was $61.2 \pm 5.6$ years old, and ranged between 53 and 72 years of age. According to TNM classification, 39 patients were in stage I, 35 patients in stage II, 41 patients in stage III, and 27 patients in stage IV lung cancer. The inclusion criteria for patients were as follows: surgery was performed for the first time, and no preoperative chemotherapy or radiation therapy was initiated. The exclusion criteria were as follows: recurrent lung cancer, previous surgeries, radiotherapy or chemotherapy treatments, other afflictions including infectious diseases, other forms of cancer, severe diabetes, severe kidney disease, pulmonary fibrosis, metabolic bone disease, systemic autoimmune disease, and cancer complications. Written informed consents were obtained from all subjects prior to surgery. We also recruited 76 non-lung cancer patients (confirmed by biopsy) who were likely to develop lung cancer (more than 50 years old, history of smoking, or family history of lung cancers) into this study. Among them, 39 were male and 37 were female. The average age of these subjects was $62.1 \pm 5.2$ years old, and ranged between 54 and 75 years of age. Lastly, 65 healthy volunteers ( 35 males and 30 females) between 52 and 76 years of age (average age $=63.2 \pm 6.2$ years) were also included in this study. There were no statistical differences in the age, gender, and clinical characteristics among all three groups. The experimental protocol was approved by the Ethics Committee of the First Affiliated Hospital of Zhengzhou University.

\section{Equipment and reagents}

Detection kits for human IL-6, IL-10, and MCP-1 were purchased from R\&D Systems (Minneapolis, MN, USA). The Labsystem Version 1.3.1 microplate reader was purchased from Bio-Rad (Hercules, CA, USA). The Elecsys automated ECL analyzer was purchased from Roche (Basel, Switzerland).

\section{Serum collection}

Fasting blood $(2 \mathrm{~mL})$ was collected from study subjects using the negative pressure collection method. Peripheral venous blood samples were collected into vacuum tubes, and were allowed coagulate at $25^{\circ} \mathrm{C}$ for $30 \mathrm{~min}$ before being centrifuged at $3600 \mathrm{rpm}$ for $10 \mathrm{~min}$ at $4^{\circ} \mathrm{C}$. Supernatants were then collected and stored at $-20^{\circ} \mathrm{C}$.

\section{Detection of CEA, CA125, and ProGRP}

Plasma levels of CEA, CA125, and ProGRP were detected using electrochemiluminescence assays. Normal values for CEA, CA125, and ProGRP were defined as $0-3.4 \mathrm{mg} / \mathrm{mL}, 0-35 \mathrm{U}$, and $0-17 \mathrm{ng} / \mathrm{mL}$, respectively.

Genetics and Molecular Research 15 (4): gmr15048949 


\section{Enzyme-linked immunosorbent assay (ELISA) for IL-6, IL-10, and MCP-1}

Plasma levels of the inflammatory factors, IL-6, IL-10, and MCP-1, were detected using ELISA, according to manufacturer protocols. In brief, $50 \mu \mathrm{L}$ stored serum samples or $50 \mu \mathrm{L}$ sequentially diluted standards were added to a 96 -well plate. Following several gentle washes, conjugated detection antibodies specific for proteins of interest $(50 \mu \mathrm{L})$ were added into each well, and the plates were incubated at $37^{\circ} \mathrm{C}$ for $30 \mathrm{~min}$. This was followed by addition of $50 \mu \mathrm{L}$ color reagent $\mathrm{A}$ and $50 \mu \mathrm{L}$ color reagent $\mathrm{B}$. The mixture was allowed to incubate in the dark at $37^{\circ} \mathrm{C}$ for $10 \mathrm{~min}$. Stop solution $(50 \mu \mathrm{L})$ was then added, and light absorbance was measured at $450 \mathrm{~nm}$.

\section{Statistical analysis}

The SPSS 16.0 software was used for statistical analyses. Measurement data were reported as means $\pm \mathrm{SD}$, and categorical data were presented as percentages. One-way analysis of variance was used when comparisons were made between multiple groups, and Fisher's least significant difference test was used to compare between two groups. Statistical significance was analyzed using chi-squared $\left(\mathrm{c}^{2}\right)$ test. $\mathrm{P}<0.05$ was considered to be statistically significant.

\section{RESULTS}

\section{Plasma levels of CEA, CA125, and ProGRP}

Serum levels of CEA, CA125, and ProGRP were measured in healthy controls, individuals with high susceptibility to lung cancer, as well as in lung cancer patients (Table 1). Our results showed that plasma CEA level was significantly higher in lung cancer patients as compared to that in healthy controls $(\mathrm{P}<0.05)$. Similarly, high-risk individuals exhibited higher serum CEA level as compared to controls, but the difference was not statistically significant. CA125 was elevated in lung cancer patients as compared to that in healthy controls and high-risk individuals $(\mathrm{P}<0.05)$. A moderate increase in CA125 was observed in the highsusceptibility group when compared to the control group. However, the difference was not statistically significant, and all CA125 values were within the normal range. ProGRP was found to be elevated in lung cancer patients as compared to that in healthy controls and highrisk individuals $(\mathrm{P}<0.05)$. Comparable ProGRP levels were found between healthy controls and high-risk individuals.

Table 1. Plasma levels of CEA, CA 125 , and ProGRP.
\begin{tabular}{l|c|c|c}
\hline & CEA $(\mathrm{ng} / \mathrm{mL})$ & CA125 $(\mathrm{U} / \mathrm{mL})$ & ProGRP $(\mathrm{ng} / \mathrm{mL})$ \\
\hline Healthy controls & $2.7 \pm 0.9$ & $22.1 \pm 3.1$ & $4.0 \pm 2.9$ \\
\hline High risk individuals & $3.6 \pm 0.3$ & $25.6 \pm 1.8$ & $5.1 \pm 1.3$ \\
\hline Pulmonary cancer patients & $5.1 \pm 0.6^{* \#}$ & $38.2 \pm 2.8^{* *}$ & $19.5 \pm 3.1^{* \#}$ \\
\hline
\end{tabular}

${ }^{*} \mathrm{P}<0.05$ compared with healthy controls; ${ }^{*} \mathrm{P}<0.05$ compared with high-risk individuals.

Serum IL-6 levels were measured via ELISA (Figure 1). Higher plasma IL-6 level was observed in lung cancer patients as compared to that in the other two groups $(\mathrm{P}<0.05)$. In addition, high-risk individuals also showed significantly elevated plasma IL-6 level as compared to the healthy controls $(\mathrm{P}<0.05)$.

Genetics and Molecular Research 15 (4): gmr15048949 


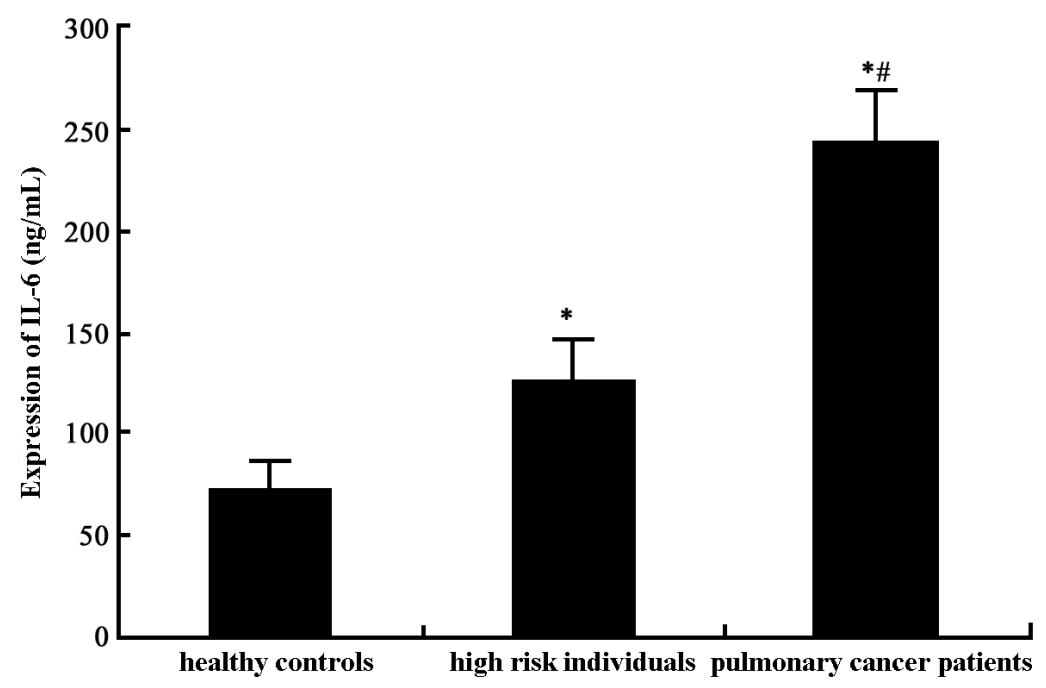

Figure 1. Serum IL-6 level. ${ }^{*} \mathrm{P}<0.05$ compared with healthy controls; ${ }^{*} \mathrm{P}<0.05$ compared with high-risk individuals.

\section{Plasma levels of IL-10}

Serum IL-10 levels were assessed using ELISA (Figure 2). Similar to IL-6, plasma IL-10 level was significantly elevated in lung cancer patients as compared to that in healthy controls and high-risk individuals $(\mathrm{P}<0.05)$. In addition, higher IL-10 level was also observed in high-risk individuals as compared to that in healthy controls $(\mathrm{P}<0.05)$.

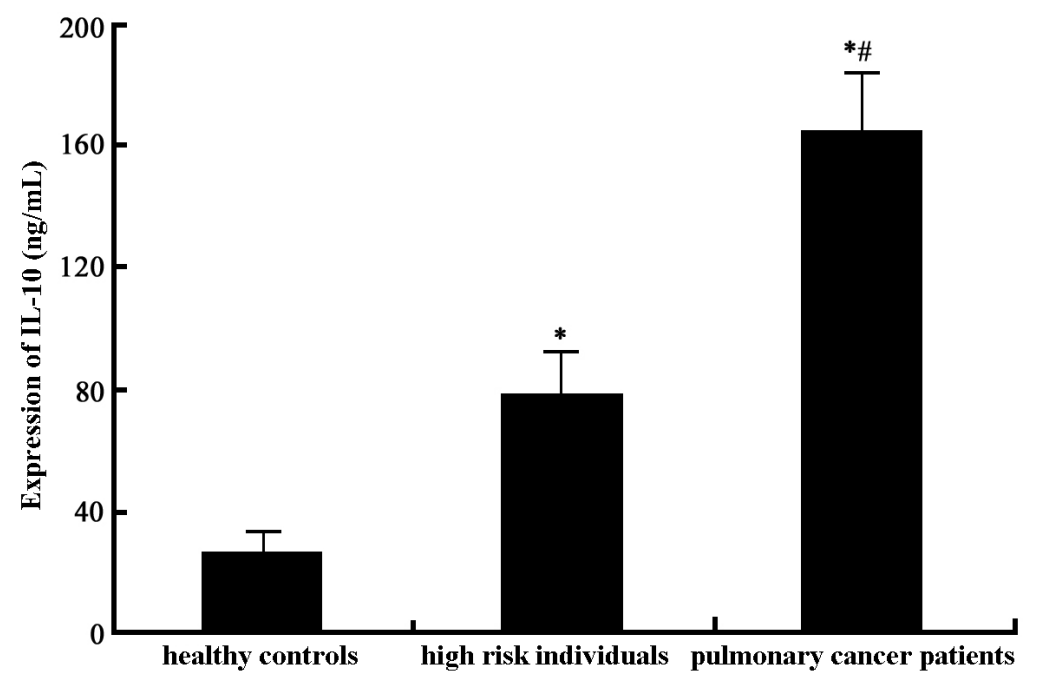

Figure 2. Plasma IL-10 level. ${ }^{*} \mathrm{P}<0.05$ compared with healthy controls; ${ }^{*} \mathrm{P}<0.05$ compared with high-risk individuals.

Genetics and Molecular Research 15 (4): gmr15048949 


\section{Plasma levels of MCP-1}

Serum IL-MCP-1 levels were assessed using ELISA (Figure 3). Our results showed that plasma MCP-1 level was significantly higher in lung cancer patients than that in healthy controls and high-risk individuals $(\mathrm{P}<0.05)$. Unlike IL-6 and IL-10, there was no statistical difference in plasma MCP-1 levels between the high-risk and control groups.

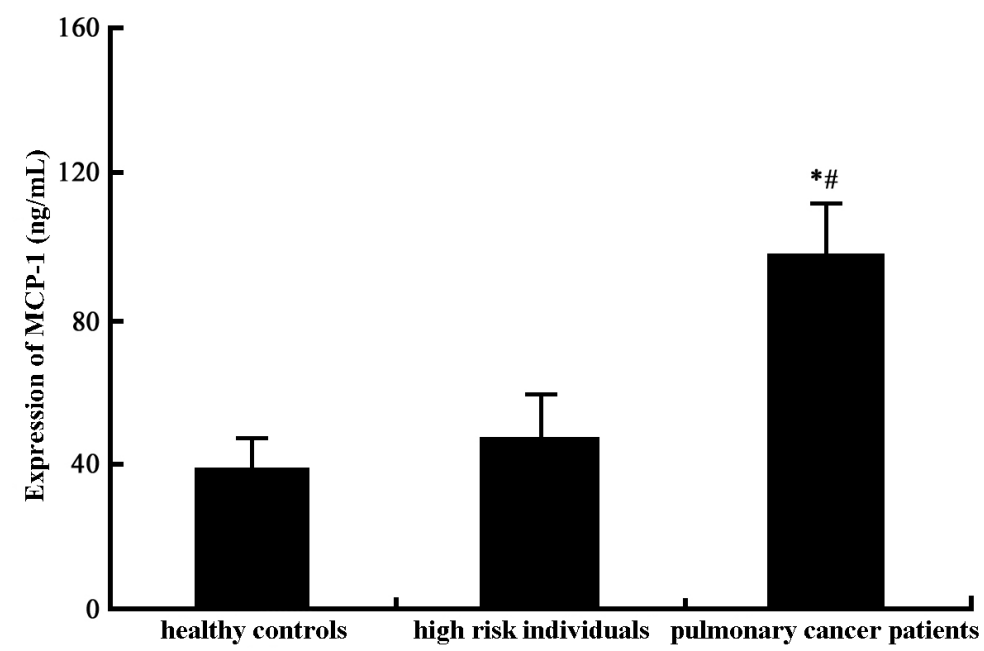

Figure 3. Plasma MCP-1 level. $* \mathrm{P}<0.05$ compared with healthy controls; ${ }^{*} \mathrm{P}<0.05$ compared with high-risk individuals.

\section{Sensitivity and specificity for lung cancer diagnosis using a single serum tumor marker}

The relationship between serum tumor markers and lung tumor detection was analyzed (Table 2). Among all markers, CA125 exhibited the highest sensitivity for lung cancer diagnosis (66.8\%), followed by CEA (55.1\%) and IL-6 (53.5\%). MCP-1 showed the least sensitivity $(38.5 \%)$ for lung tumor detection. In addition, CEA showed the highest diagnosis specificity (94.5\%), while MCP-1 was found to be the least specific (89.3\%). However, no significant difference in specificity of lung tumor detection was observed between any of the markers.

Table 2. Sensitivity and specificity in pulmonary cancer detection when using a single serum tumor marker (\%).

\begin{tabular}{l|c|c}
\hline Serum tumor marker & Sensitivity & Specificity \\
\hline CEA & 55.1 & 94.5 \\
\hline CA125 & 66.8 & 92.1 \\
\hline ProGRP & 43.7 & 90.5 \\
\hline IL-6 & 53.5 & 93.4 \\
\hline IL-10 & 49.3 & 91.3 \\
\hline MCP-1 & 38.5 & 89.3 \\
\hline
\end{tabular}

Sensitivity and specificity for lung cancer diagnosis using combined serum tumor markers

As displayed in Table 3, when all six markers were used in combination, diagnosis sensitivity reached 95.2\%. Amongst all five-marker combinations, IL-6, IL-10, CEA, CA125,

Genetics and Molecular Research 15 (4): gmr15048949 
and ProGRP displayed the highest sensitivity (92.6\%). Amongst all four-marker permutations, the combination of IL-6, CEA, CA125, and ProGRP was found to be the most sensitive $(88.6 \%)$. Furthermore, highest diagnosis specificity was achieved when all six markers were used (98.6\%); lowest specificity was found with three-marker combinations.

\begin{tabular}{|c|c|c|}
\hline Serum tumor marker combinations & Sensitivity & Specificity \\
\hline CEA + CA125 + ProGRP & 75.2 & 96.1 \\
\hline CEA + CA125 + ProGRP + IL-6 & 88.6 & 96.9 \\
\hline CEA + CA125 + ProGRP + IL-10 & 84.5 & 97.1 \\
\hline CEA + CA125 + ProGRP + MCP-1 & 80.2 & 96.2 \\
\hline $\mathrm{CEA}+\mathrm{CA} 125+$ ProGRP + IL-6 + IL-10 & 92.6 & 97.8 \\
\hline CEA + CA125 + ProGRP + IL-6 + MCP-1 & 90.7 & 97.5 \\
\hline CEA + CA125 + ProGRP + IL-10 + MCP-1 & 89.2 & 97.3 \\
\hline CEA + CA125 + ProGRP + IL-6 + IL-10 + MCP-1 & 95.2 & 98.6 \\
\hline
\end{tabular}

\section{DISCUSSION}

The onset and development of lung cancer is dependent on multiple factors, and is the result of various signaling pathways controlled by an array of genes. The lack of rapid and accurate methods for early diagnosis of lung cancer, together with ineffective treatments for advanced stages of lung cancer, all lead to poor disease prognosis. In previous years, the most common diagnostic tools for lung cancer included CT, X-chip imaging, and sputum cytology examination. However, these methods lack sensitivity and specificity, and often lead to misdiagnosis (Sasaki et al., 2016; Zaric et al., 2016). Currently, medical imaging, bronchoscopy, bronchial perfusion fluid examinations, and tissue pathology analyses are widely used in lung cancer screening and early diagnosis (Huang et al., 2016). Understanding the biological characteristics and molecular mechanisms of lung cancer has facilitated the development of new diagnostic tools and treatments for this disease. However, there are no recognized clinical indicators for lung cancer prevention, early diagnosis, and treatment. Therefore, early disease detection, improvement in treatment and prognosis, decrease in lung cancer incidence and mortality rates, as well as increase in survival rates are all important issues to tackle (Ortakoylu et al., 2015; Xiao et al., 2015).

Serum tumor markers are proteins or enzymes synthesized in large quantities by tumor cells, and they are released into the blood during tumor onset and development. In lung cancers, abnormal expression of serum tumor markers precedes typical clinical symptoms (cough, expectoration, bloody sputum, pain, and pleural effusion). Therefore, detection of plasma tumor markers is vital for screening and early diagnosis of lung cancer (Chen et al., 2015a). The most commonly used serum tumor markers of lung cancer include CEA, CA125, and ProGRP. CEA is a tumor-associated antigen with specific embryonic antigen determinants; up-regulation of CEA is not lung cancer-specific. It is widely expressed in a variety of tumors (digestive tract cancer, breast cancer, and urogenital tract cancer) and in pneumonia patients. CA125 has been found to be mainly present in ovarian cancer patients, but also has diagnostic values for lung cancer. ProGRP has been shown to be especially sensitive for detecting small-cell lung cancer, non-small cell lung cancer, and metastatic lung cancer. The specificity and sensitivity of lung cancer detection is limited when these three markers are used separately, with CA125 being the best predictor for lung cancer $(66.8 \%)$. When used

Genetics and Molecular Research 15 (4): gmr15048949 
in combination, diagnosis sensitivity is increased to $75.2 \%$. It is, therefore, critical to identify more specific and sensitive serum markers for lung cancer. It has been shown that IL-6, IL-10, and MCP-1 are up-regulated in lung cancer patients (Lan et al., 2015; Shinke et al., 2015). In this study, we found that MCP-1 expression was significantly higher in lung cancer patients as compared to that in high risk and healthy individuals. Furthermore, expressions of IL-6 and IL-10 were both elevated in lung cancer patients and high-risk individuals as compared to that in healthy controls. We also examined the effect of combination serum markers on sensitivity and specificity of lung cancer detection. Our results showed that greatest sensitivity was achieved when all six markers were used during analysis. This was followed by a five-marker combination using IL-6, IL-10, CEA, CA125, and ProGRP. In terms of fourmarker combinations, analysis using IL-6, CEA, CA125, and ProGRP was found to be the most sensitive. Similar to sensitivity results, six-marker combinations resulted in the highest specificity, while three-marker combinations demonstrated the lowest specificity. However, no significant difference in specificity was found amongst the different combinations. These results demonstrated that the combination of tumor-related inflammatory molecules (IL-6, IL10, and MCP-1) with traditional serum tumor markers (CEA, CA125, and ProGRP) resulted in more accurate lung cancer diagnosis. However, the exact mechanism of IL-6, IL-10, and MCP-1 in lung cancer pathophysiology is still unclear. Recent evidence suggests that IL- 6 and MCP-1 genetic polymorphism is associated with prognosis and susceptibility of non-small cell lung cancer (Yang et al., 2010; Zhao et al., 2016). Therefore, extensive studies on the feasibility of using inflammatory factors as tumor markers in a large cohort of patients with diverse genetic backgrounds should be validated in the future.

In summary, this study showed that the tumor markers, CEA, CA125, and ProGRP, in combination with IL-6, IL-10, and MCP-1, yielded better lung cancer diagnosis. This method can be used for screening and diagnosis of early stages of lung cancer, and it may improve prognosis and treatment effects in lung cancer patients.

\section{Conflicts of interest}

The authors declare no conflict of interest.

\section{ACKNOWLEDGMENTS}

Research supported by the National Natural Science Fund of China (Grant\#81301220).

\section{REFERENCES}

Bhora FY, Chen DJ, Detterbeck FC, et al. (2014). The ITMIG/IASLC thymic epithelial tumors staging project: a proposed lymph node map for thymic epithelial tumors in the forthcoming 8th edition of the TNM classification of malignant tumors. J. Thorac. Oncol. 9: S88-96.

Burns TF and Stabile LP (2014). Targeting the estrogen pathway for the treatment and prevention of lung cancer. Lung Cancer Manag. 3: 43-52. http://dx.doi.org/10.2217/lmt.13.67

Chen F, Yan CE, Li J, Han XH, et al. (2015a). Diagnostic value of CYFRA 21-1 and CEA for predicting lymph node metastasis in operable lung cancer. Int. J. Clin. Exp. Med. 8: 9820-9824.

Chen Y, Zhang F, Tsai Y, Yang X, et al. (2015b). IL-6 signaling promotes DNA repair and prevents apoptosis in CD133+ stem-like cells of lung cancer after radiation. Radiat. Oncol. 10: 227. http://dx.doi.org/10.1186/s13014-015-0534-1

Fiala O, Pesek M, Finek J, Svaton M, et al. (2016). Prognostic significance of serum tumor markers in patients with advanced-stage NSCLC treated with pemetrexed-based chemotherapy. Anticancer Res. 36: 461-466.

Genetics and Molecular Research 15 (4): gmr15048949 
Genestreti G, Grossi F, Genova C, Burgio MA, et al. (2014). Third- and further-line therapy in advanced non-small-cell lung cancer patients: an overview. Future Oncol. 10: 2081-2096. http://dx.doi.org/10.2217/fon.14.96

Hu L, Ibrahim S, Liu C, Skaar J, et al. (2009). Thrombin induces tumor cell cycle activation and spontaneous growth by down-regulation of p27Kip1, in association with the up-regulation of Skp2 and MiR-222. Cancer Res. 69: 33743381. http://dx.doi.org/10.1158/0008-5472.CAN-08-4290

Huang Z, Yi X, Luo B, Zhu J, et al. (2016). Induced sputum deposition improves diagnostic yields of pulmonary alveolar proteinosis: A clinicopathological and methodological study of 17 cases. Ultrastruct. Pathol. 40: 7-13. http://dx.doi. org $/ 10.3109 / 01913123.2015 .1104404$

Lan X, Lan T and Faxiang Q (2015). Interleukin-10 promoter polymorphism and susceptibility to lung cancer: a systematic review and meta-analysis. Int. J. Clin. Exp. Med. 8: 15317-15328.

Ma L, Xie XW, Wang HY, Ma LY, et al. (2015). Clinical evaluation of tumor markers for diagnosis in patients with non-small cell lung cancer in China. Asian Pac. J. Cancer Prev. 16: 4891-4894. http://dx.doi.org/10.7314/ APJCP.2015.16.12.4891

Northcott PA, Fernandez-L A, Hagan JP, Ellison DW, et al. (2009). The miR-17/92 polycistron is up-regulated in sonic hedgehog-driven medulloblastomas and induced by N-myc in sonic hedgehog-treated cerebellar neural precursors. Cancer Res. 69: 3249-3255. http://dx.doi.org/10.1158/0008-5472.CAN-08-4710

Ortakoylu MG, Iliaz S, Bahadir A, Aslan A, et al. (2015). Diagnostic value of endobronchial ultrasound-guided transbronchial needle aspiration in various lung diseases. J. Bras. Pneumol. 41: 410-414. http://dx.doi.org/10.1590/ $\underline{\text { S1806-37132015000004493 }}$

Pang JC, Kwok WK, Chen Z and Ng HK (2009). Oncogenic role of microRNAs in brain tumors. Acta Neuropathol. 117: 599-611. http://dx.doi.org/10.1007/s00401-009-0525-0

Qader AA, Urraca J, Torsetnes SB, Tønnesen F, et al. (2014). Peptide imprinted receptors for the determination of the small cell lung cancer associated biomarker progastrin releasing peptide. J. Chromatogr. A 1370: 56-62. http:// dx.doi.org/10.1016/j.chroma.2014.10.023

Reddy SP, Natarajan V and Dudek AZ (2014). MARCKS is marked in combating lung cancer growth and acquired resistance. Am. J. Respir. Crit. Care Med. 190: 1084-1086. http://dx.doi.org/10.1164/rccm.201410-1922ED

Sasaki S, Yoshioka Y, Ko R, Katsura Y, et al. (2016). Diagnostic significance of cerebrospinal fluid EGFR mutation analysis for leptomeningeal metastasis in non-small-cell lung cancer patients harboring an active EGFR mutation following gefitinib therapy failure. Respir. Investig. 54: 14-19. http://dx.doi.org/10.1016/j.resinv.2015.07.001

Shi P, Meng X, Ni M, Sun X, et al. (2015). Association between serum tumor markers and metabolic tumor volume or total lesion glycolysis in patients with recurrent small cell lung cancer. Oncol. Lett. 10: 3123-3128.

Shinke H, Masuda S, Togashi Y, Ikemi Y, et al. (2015). Urinary kidney injury molecule-1 and monocyte chemotactic protein-1 are noninvasive biomarkers of cisplatin-induced nephrotoxicity in lung cancer patients. Cancer Chemother. Pharmacol. 76: 989-996. http://dx.doi.org/10.1007/s00280-015-2880-y

Xiao H, Liu Y, Tan H, Liang P, et al. (2015). A pilot study using low-dose Spectral CT and ASIR (Adaptive Statistical Iterative Reconstruction) algorithm to diagnose solitary pulmonary nodules. BMC Med. Imaging 15: 54. http:// dx.doi.org/10.1186/s12880-015-0096-6

Yang J, Wei F, Schafer C and Wong DT (2014). Detection of tumor cell-specific mRNA and protein in exosome-like microvesicles from blood and saliva. PLoS One 9: e110641. http://dx.doi.org/10.1371/journal.pone.0110641

Yang L, Shi GL, Song CX and Xu SF (2010). Relationship between genetic polymorphism of MCP-1 and non-small-cell lung cancer in the Han nationality of North China. Genet. Mol. Res. 9: 765-771. http://dx.doi.org/10.4238/vol9$\underline{\operatorname{gmmr} 740}$

Zaric B, Stojsic V, Carapic V, Kovacevic T, et al. (2016). Radial endobronchial ultrasound (EBUS) guided suction catheterbiopsy in histological diagnosis of peripheral pulmonary lesions. J. Cancer 7: 7-13. http://dx.doi.org/10.7150/ jca. 13081

Zhao K, Xu J and Tian H (2016). Correlation analysis between an IL-6 genetic polymorphism and non-small cell lung cancer prognosis. Genet. Mol. Res. 15: 15017021. http://dx.doi.org/10.4238/gmr.15017021

Genetics and Molecular Research 15 (4): gmr15048949 